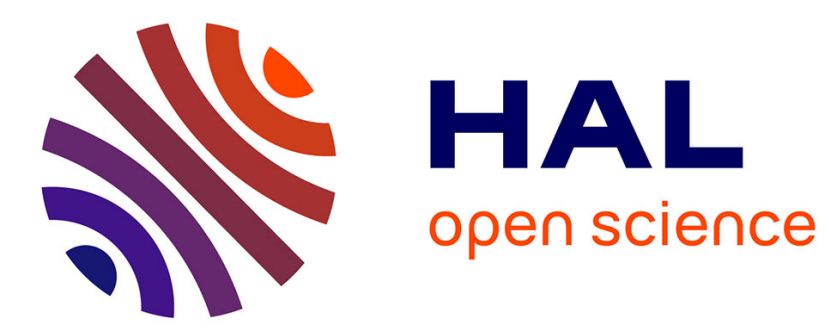

\title{
Miming the cancer-immune system competition by kinetic Monte Carlo simulations
}

Carlo Bianca, Annie Lemarchand

\section{To cite this version:}

Carlo Bianca, Annie Lemarchand. Miming the cancer-immune system competition by kinetic Monte Carlo simulations. Journal of Chemical Physics, 2016, 145 (15), pp.154108. 10.1063/1.4964778 . hal-01386750

\section{HAL Id: hal-01386750 \\ https://hal.sorbonne-universite.fr/hal-01386750}

Submitted on 24 Oct 2016

HAL is a multi-disciplinary open access archive for the deposit and dissemination of scientific research documents, whether they are published or not. The documents may come from teaching and research institutions in France or abroad, or from public or private research centers.
L'archive ouverte pluridisciplinaire HAL, est destinée au dépôt et à la diffusion de documents scientifiques de niveau recherche, publiés ou non, émanant des établissements d'enseignement et de recherche français ou étrangers, des laboratoires publics ou privés. 


\section{AlP | $\begin{aligned} & \text { The Journal of } \\ & \text { Chemical Physics }\end{aligned}$}

\section{Miming the cancer-immune system competition by kinetic Monte Carlo simulations}

Carlo Bianca and Annie Lemarchand

Citation: The Journal of Chemical Physics 145, 154108 (2016); doi: 10.1063/1.4964778

View online: http://dx.doi.org/10.1063/1.4964778

View Table of Contents: http://scitation.aip.org/content/aip/journal/jcp/145/15?ver=pdfcov

Published by the AIP Publishing

\section{Articles you may be interested in}

Periodic and chaotic oscillations in a tumor and immune system interaction model with three delays

Chaos 24, 023101 (2014); 10.1063/1.4870363

Development of a randomized 3D cell model for Monte Carlo microdosimetry simulations

Med. Phys. 39, 3509 (2012); 10.1118/1.4719963

Macroion solutions in the cell model studied by field theory and Monte Carlo simulations

J. Chem. Phys. 135, 224508 (2011); 10.1063/1.3665450

Monte Carlo electron source model validation for an Elekta Precise linac

Med. Phys. 38, 2366 (2011); 10.1118/1.3570579

Improving IMRT dose accuracy via deliverable Monte Carlo optimization for the treatment of head and neck cancer patients

Med. Phys. 33, 4033 (2006); 10.1118/1.2357835

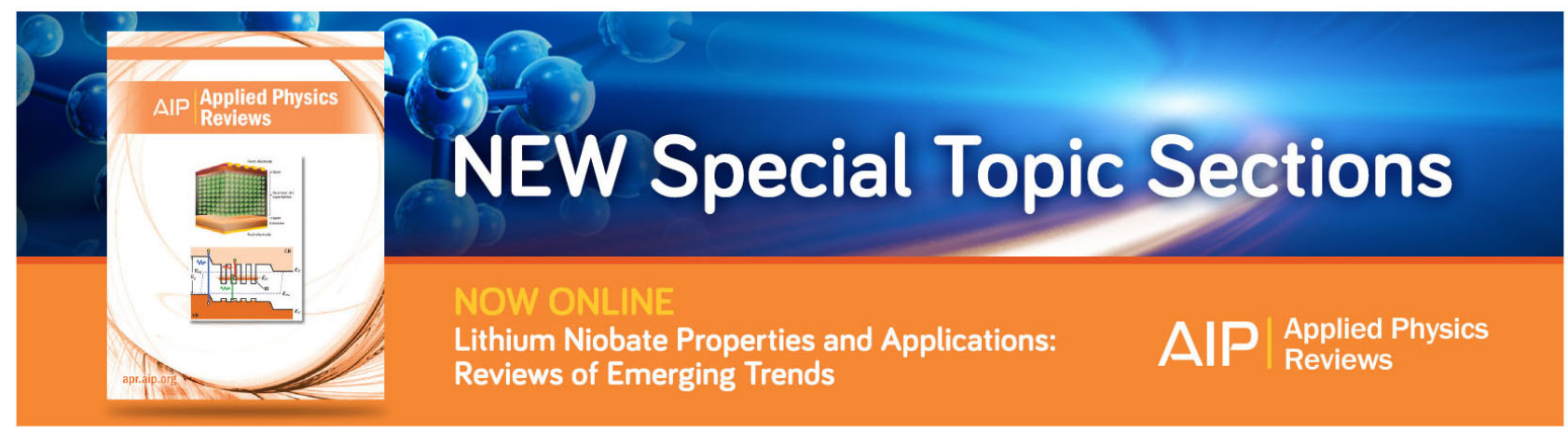




\title{
Miming the cancer-immune system competition by kinetic Monte Carlo simulations
}

\author{
Carlo Bianca ${ }^{1}$ and Annie Lemarchand 2,3, a) \\ ${ }^{1}$ Sorbonne Universités, UPMC Univ Paris 06, CNRS, Univ Paris Diderot, Ecole Normale Supérieure \\ Laboratoire de Physique Statistique, 24 Rue Lhomond, 75005 Paris, France \\ ${ }^{2}$ Sorbonne Universités, UPMC Univ Paris 06, Laboratoire de Physique Théorique de la Matière Condensée \\ (LPTMC), 4 Place Jussieu, Case Courrier 121, 75252 Paris Cedex 05, France \\ ${ }^{3}$ CNRS, LPTMC UMR 7600, Paris, France
}

(Received 22 July 2016; accepted 30 September 2016; published online 18 October 2016)

\begin{abstract}
In order to mimic the interactions between cancer and the immune system at cell scale, we propose a minimal model of cell interactions that is similar to a chemical mechanism including autocatalytic steps. The cells are supposed to bear a quantity called activity that may increase during the interactions. The fluctuations of cell activity are controlled by a so-called thermostat. We develop a kinetic Monte Carlo algorithm to simulate the cell interactions and thermalization of cell activity. The model is able to reproduce the well-known behavior of tumors treated by immunotherapy: the first apparent elimination of the tumor by the immune system is followed by a long equilibrium period and the final escape of cancer from immunosurveillance. Published by AIP Publishing. [http://dx.doi.org/10.1063/1.4964778]
\end{abstract}

\section{INTRODUCTION}

The activation and response of the immune system to antigens is a complex process which involves different cells and molecules. In particular the immune system response to mutated cells, and more specifically to cancer cells, has gained much attention at the beginning of the twentieth century. ${ }^{1}$ However, a minimal interpretation of tumor immunogenicity, according to which a tumor induces a response of the immune system that is able to eliminate the tumor, has proven insufficient. More complex interactions between the cancer cells and the immune system cells need to be considered to account for tumor development in the presence of antigens. ${ }^{2,3}$ The treatment of cancer by immunotherapy and the development of successful vaccination protocols require a refinement of the understanding of cancer immunosurveillance. The concept of immunoediting has been introduced in order to take account of the dual role of the immune system in both the elimination of cancer and the possible promotion of tumor growth., Immunoediting is supposed to encompass three processes, elimination, equilibrium, and escape, known as the three E's. ${ }^{2}$ After a first step of tumor elimination, during which the immune system successfully controls the tumor, the immune system itself is suspected of promoting the generation of tumor cell variants able to resist to the attack of the immune cells. This second step is called equilibrium, due to the apparent stationary state of the system. However, a third step of tumor escape from immune destruction can be then observed.

The aim of the present paper is to develop a kinetic Monte Carlo approach in order to simulate the interactions

\footnotetext{
a) Author to whom correspondence should be addressed. Electronic mail: anle@1ptmc.jussieu.fr
}

between the tumor and immune system at the cell scale. We recently developed a kinetic theory approach to cell interactions $^{6-8}$ relying on hypotheses that are not easily testable for the moment. As far-from-equilibrium living units, cells are supposed to follow a strategy that can be modeled by the tendency to increase a nonconserved, scalar quantity, further referred to as activity. This quantity should not be confused with the effective concentration of a chemical species in a mixture. The term has been chosen in reference to active matter, ${ }^{9}$ a system composed of a large number of agents which are able to produce work at the expense of energy consumption. In the present context, activity reflects cell heterogeneity due to mutations. For immune system cells, activity captures the level of learning reached at the contact with cancer cells through the recognition process. The activity allocated to cancer cells represents the progression towards high values of malignancy. The considered system is spatially homogeneous but the model mimics the biological heterogeneity of cells by initially allocating different activities to the cells and controlling the degree of heterogeneity by the width of the probability distribution of the activity. We design a model of binary cell interactions, considered as Markov processes with activity-dependent transition rates. The binary interactions lead to cell mutation and tend to increase the activity of a cell. Exchanges with the microenvironment of the cell are supposed to act as a bath for activity fluctuations. By analogy with the control of energy fluctuations, the term of thermostat is used to describe the control of the second moment of the activity. ${ }^{10,11}$

The kinetic theory was originally designed to describe molecular collisions in dilute gases. ${ }^{12}$ Applications to physical systems include the modeling of dissipative interactions between larger particles, in particular in the case of granular flows. ${ }^{13-15}$ The kinetic equations are nonlinear partial integro- 
differential equations that can be numerically solved using a direct simulation of the underlying stochastic processes according to kinetic Monte Carlo approaches. ${ }^{16,17}$ The direct simulation Monte Carlo (DSMC) method ${ }^{16,18}$ has been introduced to simulate rare events in dilute gases and is in particular well suited for the simulation of exothermic reactions at large Knudsen number. ${ }^{19,20}$ However, the scope of the method has been extended to soft matter and DSMC has been successfully used to simulate phenomena of biological relevance such as somitogenesis ${ }^{21}$ and chemotaxis. ${ }^{22}$ In the case of cell interactions, we propose an algorithm based on a succession of randomly chosen binary interactions and thermalization steps. The possibility of reproducing classical pathological cell behaviors can be envisaged as an indirect, partial proof of our kinetic theory approach to cell interactions.

The paper is organized as follows. The model of cell interactions is described in Section II and the kinetic equations for the distribution functions of the different types of cells are derived. The kinetic Monte Carlo algorithm is made precise in Section III. The results are discussed in Section IV. In particular the ability of the model to take into account the three E's of cancer immunoediting is examined and special attention is devoted to the action of the thermostat. Section V contains conclusions.

\section{MODEL}

We consider a spatially homogeneous system composed by three different cell populations: cancer cells $c$, immune system cells $i$, and normal cells $n$. The initial state of the system is defined by the numbers $N_{c}, N_{i}$, and $N_{n}$ of cells of each population $c, i$, and $n$, respectively. The system is open and in contact with a source $S$ of normal cells which maintains the number $N_{n}$ of normal cells constant. An activity variable, $u \in \mathbb{R}^{+}$, initially distributed according to a normal distribution, $P(u)=\frac{1}{\sigma \sqrt{2 \pi}} \mathrm{e}^{-\left(\frac{u-\mu}{\sigma \sqrt{2}}\right)^{2}}$, of mean value $\mu$ and standard deviation $\sigma$, biased by imposing $u \geq 0$, is allocated to each cell. We introduce a minimal model which considers the onset of cell mutation. The model is based on binary interactions among the different types of cell populations. The magnitude of the activity increases through the interactions.

The model is based on the following assumptions. A cancer cell $c(u)$ of activity $u$ is able to mutate a normal cell $n\left(u^{\prime}\right)$ of smaller activity $u^{\prime}<u$ into a cancer cell $c\left(u^{\prime}\right)$ at an activity-dependent rate and, consequently, to increase its own activity by a small amount, $\epsilon$. At the same rate, the source $S$ injects a normal cell $n\left(u^{\prime \prime}\right)$ with a normally distributed activity $u^{\prime \prime}$ so that the number $N_{n}$ of normal cells remains constant

$$
\left\{\begin{array}{l}
c(u)+n\left(u^{\prime}\right) \stackrel{k_{1}\left(u-u^{\prime}\right) H\left(u-u^{\prime}\right)}{\longrightarrow} c(u+\epsilon)+c\left(u^{\prime}\right), \\
S \longrightarrow n\left(u^{\prime \prime}\right)
\end{array}\right.
$$

where $H(u)$ is the Heaviside step function and $k_{1}$ is a rate constant. By analogy with a chemical mechanism, Eq. (1) can be viewed as an autocatalytic production of cancer cells, in so far as cancer cells are both reactants and products of the interaction.

In order to take into account the dual role of the immune system in fighting cancer and promoting it, we introduce two different types of interactions between cancer cells and immune system cells. Depending on the values of their activity, the interaction between a cancer cell $c$ and an immune system cell $i$ may lead to either two immune system cells or two cancer cells

$$
\begin{aligned}
& c(u)+i\left(u^{\prime}\right) \stackrel{k_{2}\left(u^{\prime}-u\right) H\left(u^{\prime}-u\right)}{\longrightarrow} i(u)+i\left(u^{\prime}+\epsilon\right), \\
& c(u)+i\left(u^{\prime}\right) \stackrel{k_{3}\left(u-u^{\prime}\right) H\left(u-u^{\prime}\right)}{\longrightarrow} c(u+\epsilon)+c\left(u^{\prime}\right),
\end{aligned}
$$

where $k_{2}$ and $k_{3}$ are rate constants. In both cases, the interaction also results in assigning an activity increased by $\epsilon$ to the cell with the largest activity before the interaction. At cell scale, the autocatalytic production of immune system cells by the process given in Eq. (2) can be related to tumor elimination, whereas the autocatalytic production of cancer cells by the process given in Eq. (3) can be related to the phenomenon of tumor escape introduced in immunoediting. ${ }^{2}$

Due to the interactions, the activity of the cells evolves and fluctuates. A thermostat associated with a field $E$ is introduced in order to control the second moment of the activity of the entire system. For a Gaussian isokinetic thermostat where the kinetic energy is conserved, the constrained equations of motion introduce a dissipative term proportional to the velocity in the equation relating acceleration and forces. By analogy with Newtonian equations, we write

$$
\frac{\mathrm{d} u}{\mathrm{~d} t}=E-\alpha u
$$

where $\alpha$ can be seen as a generalized coefficient of friction. Imposing that the second moment $\left\langle u^{2}\right\rangle$ of the activity is conserved leads to $\left\langle u \frac{\mathrm{d} u}{\mathrm{~d} t}\right\rangle=0=\langle u\rangle E-\alpha\left\langle u^{2}\right\rangle$, which results in

$$
\alpha=\frac{\langle u\rangle E}{\left\langle u^{2}\right\rangle} .
$$

In Eqs. (4) and (5), $\langle$.$\rangle has to be interpreted as an ensemble$ average.

In the framework of kinetic theory, distribution functions $f_{j}(t, u)$ depending on time and activity for each kind of cells $j=n, i, c$ are introduced. According to the thermostatted kinetic approach introduced in Refs. 6-8, the time evolution of the distribution functions $f_{j}(t, u)$ can be written as

$$
\partial_{t} f_{j}(t, u)+\partial_{u}\left(F(u) f_{j}\right)=I_{j},
$$

where the term $F(u)$ refers to the thermostat and the term $I_{j}$ is the interaction term affecting the cells of type $j$ and resulting from the processes given in Eqs. (1)-(3). Specifically, the interaction term $I_{c}$ associated with the cancer cells reads 


$$
\begin{aligned}
I_{c}= & \int_{\mathbb{R}^{+}} k_{1}\left(u-\epsilon-u^{\prime}\right) H\left(u-\epsilon-u^{\prime}\right) f_{c}(t, u-\epsilon) f_{n}\left(t, u^{\prime}\right) \mathrm{d} u^{\prime} \\
& +\int_{\mathbb{R}^{+}} k_{1}\left(u^{\prime}-u\right) H\left(u^{\prime}-u\right) f_{c}\left(t, u^{\prime}\right) f_{n}(t, u) \mathrm{d} u^{\prime} \\
& -\int_{\mathbb{R}^{+}} k_{2}\left(u^{\prime}-u\right) H\left(u^{\prime}-u\right) f_{c}(t, u) f_{i}\left(t, u^{\prime}\right) \mathrm{d} u^{\prime} \\
& +\int_{\mathbb{R}^{+}} k_{3}\left(u-\epsilon-u^{\prime}\right) H\left(u-\epsilon-u^{\prime}\right) f_{c}(t, u-\epsilon) f_{i}\left(t, u^{\prime}\right) \mathrm{d} u^{\prime} \\
& +\int_{\mathbb{R}^{+}} k_{3}\left(u^{\prime}-u\right) H\left(u^{\prime}-u\right) f_{c}\left(t, u^{\prime}\right) f_{i}(t, u) \mathrm{d} u^{\prime},
\end{aligned}
$$

where the first and second integrals refer to the autocatalytic generation of cancer cells by Eq. (1), the third integral refers to the destruction of cancer cells by Eq. (2), and the fourth and fifth integrals refer to the autocatalytic production of cancer cells by Eq. (3). Similarly, the interaction term $I_{i}$ associated with the immune system cells is

$$
\begin{aligned}
I_{i}= & \int_{\mathbb{R}^{+}} k_{2}\left(u-\epsilon-u^{\prime}\right) H\left(u-\epsilon-u^{\prime}\right) f_{c}\left(t, u^{\prime}\right) \\
& \times f_{i}(t, u-\epsilon) \mathrm{d} u^{\prime} \\
& +\int_{\mathbb{R}^{+}} k_{2}\left(u^{\prime}-u\right) H\left(u^{\prime}-u\right) f_{c}(t, u) f_{i}\left(t, u^{\prime}\right) \mathrm{d} u^{\prime} \\
& -\int_{\mathbb{R}^{+}} k_{3}\left(u^{\prime}-u\right) H\left(u^{\prime}-u\right) f_{c}\left(t, u^{\prime}\right) f_{i}(t, u) \mathrm{d} u^{\prime},
\end{aligned}
$$

where the first and second integrals are related to the autocatalytic production of immune system cells due to the process given in Eq. (2) and the third integral is related to tumor counterattack of immune system cells according to Eq. (3). Finally, the interaction term $I_{n}$ for the normal cells is given by

$$
\begin{aligned}
I_{n}= & -\int_{\mathbb{R}^{+}} k_{1}\left(u^{\prime}-u\right) H\left(u^{\prime}-u\right) f_{c}\left(t, u^{\prime}\right) f_{n}(t, u) \mathrm{d} u^{\prime} \\
& +P(u) \int_{\mathbb{R}^{+}} \int_{\mathbb{R}^{+}} k_{1}\left(u^{\prime}-u\right) H\left(u^{\prime}-u^{\prime \prime}\right) f_{c}\left(t, u^{\prime}\right) \\
& \times f_{n}\left(t, u^{\prime \prime}\right) \mathrm{d} u^{\prime} \mathrm{d} u^{\prime \prime},
\end{aligned}
$$

where the first integral originates from the mutation of normal cells by the process given in Eq. (1) and the second integral accounts for the effect of the source of normal cells with activities distributed according to the normalized distribution $P(u)$. By integrating Eq. (6) over $u$ for $j=n$, we straightforwardly get $\partial_{t} \int_{\mathbb{R}^{+}} f_{n}(t, u) \mathrm{d} u=0$ and check that the density $\rho_{n}=\int_{\mathbb{R}^{+}} f_{n}(t, u) d u$ of normal cells is actually kept constant.

Due to the mutation of normal cells into cancer cells and the simultaneous re-injection of normal cells into the system through the process given in Eq. (1), the total number of cells increases. Hence, the sum of the interaction terms does not vanish

$$
\sum_{j=n, i, c} I_{j} \neq 0
$$

and the second moment of the activity,

$$
\left\langle u^{2}\right\rangle=\int_{\mathbb{R}^{+}} u^{2} \sum_{j=n, i, c} f_{j}(t, u) \mathrm{d} u,
$$

is not strictly conserved. However, in order to prevent an explosion of activity fluctuations and for the sake of simplicity, we introduce the same thermostat, as if the total number of cells was conserved. By analogy with the coefficient of friction $\alpha$ introduced in Eqs. (4) and (5), we look for a thermostat term in the form $F(u)=E-\alpha u$ in Eq. (6) and obtain

$$
F(u)=E\left[1-u \int_{\mathbb{R}^{+}} u\left(\sum_{j=n, i, c} f_{j}(t, u)\right) \mathrm{d} u\right] .
$$

In Sec. III, we propose to directly simulate the stochastic processes defined in Eqs. (1)-(3) in order to solve numerically the nonlinear partial integro-differential kinetic equations (Eqs. (6)-(9)) which govern the far-fromequilibrium dynamics of the cancer and immune system competition.

\section{KINETIC MONTE CARLO ALGORITHM}

Kinetic Monte Carlo methods are designed to simulate stochastic processes whose transition rates are known. In particular, the algorithm introduced by Gillespie is used to numerically solve a master equation associated with reactiondiffusion processes. ${ }^{17}$ The master equation is intrinsically stochastic. It has the form of a unique, linear equation for a probability distribution. ${ }^{23}$ Without the thermostat, it would have been possible to write a master equation associated with the cell interactions defined in Eqs. (1)-(3). The probability would have been a function of the activities and numbers of cells of each type, considered as discrete, random variables. We rather opted for an approach in the framework of kinetic theory, first to easily control activity fluctuations and second with the future aim of introducing spatial homogeneities, i.e., following the distributions of cell position and velocity. ${ }^{8}$ There are significant differences with the master equation approach. It is necessary to write as many kinetic equations as cell types. The interaction terms given in Eqs. (7)-(9) are nonlinear functions of the distribution functions. The kinetic equations involve continuous distribution functions. Their integration over the activity $u$ provides deterministic densities for each cell type.

Bird $^{16}$ proposed an efficient kinetic Monte Carlo algorithm to solve the Boltzmann equation associated with a dilute gas. The direct simulation Monte Carlo (DSMC) method $^{16,18}$ introduces effective particles and generates their stochastic trajectories. Consequently, DSMC includes the description of the fluctuations of particle numbers, ${ }^{24}$ in common with the master equation. The direct simulation Monte Carlo method even gives access to the dynamics of each particle, whereas the Gillespie algorithm only provides the evolution of particle numbers: DSMC is a particle dynamics simulation method but the master equation and Gillespie algorithm describe the system at a mesoscopic scale. After ensemble averaging, it has been proven that DSMC gives an exact solution of the Boltzmann equation. ${ }^{25}$ In order to simulate cell interactions, we have adapted the standard acceptance-rejection technique used in DSMC as follows. The particles are cells. Each cell has an activity $u$. Time is discretized. The time step $\Delta t$ is constant, contrary to the waiting time for the next random process in the Gillespie algorithm. During the time step $\Delta t$, interactions between 


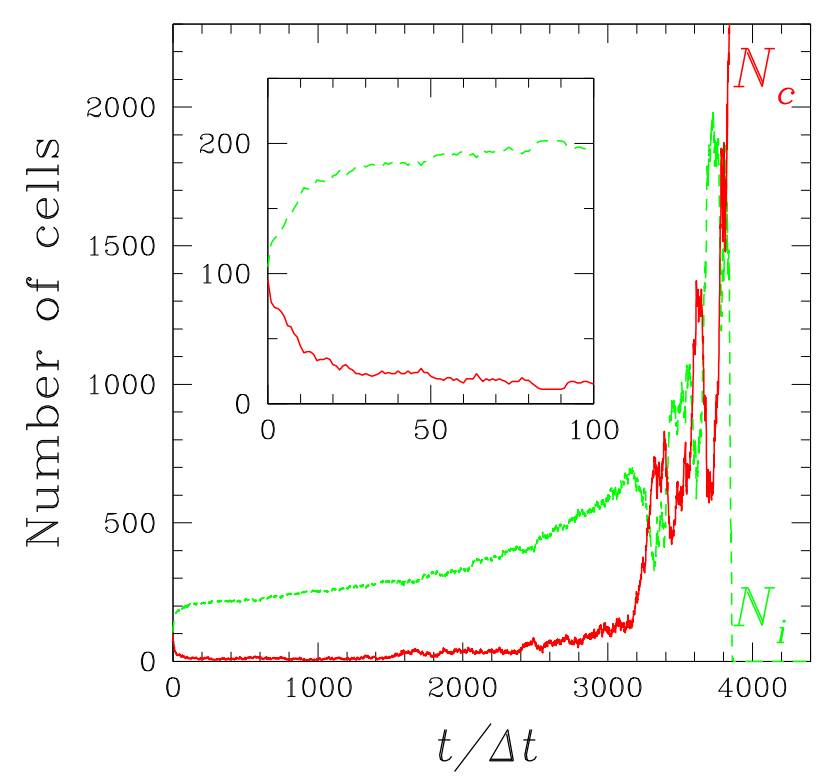

(a)

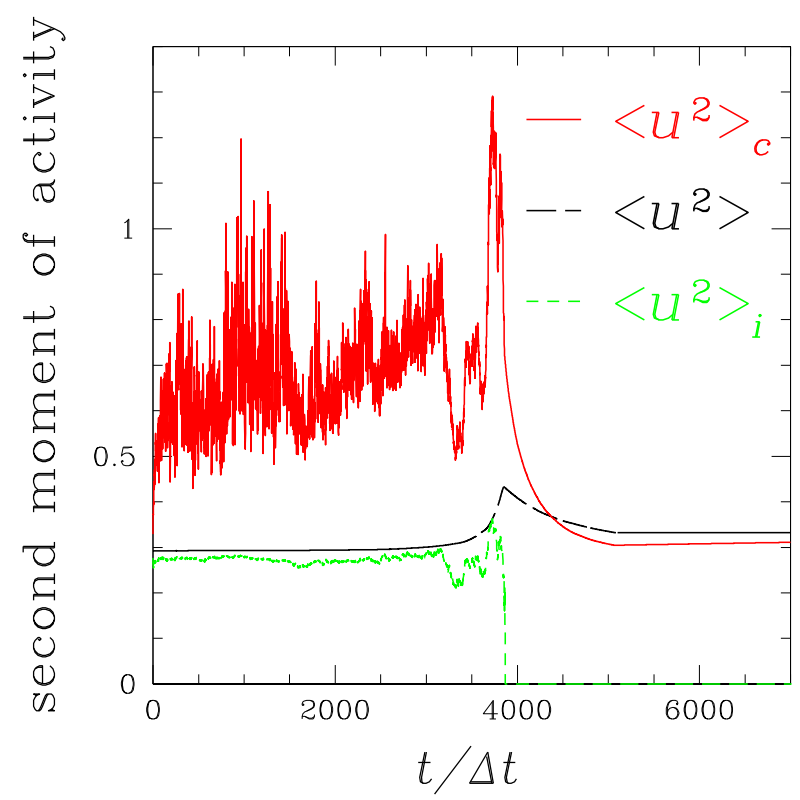

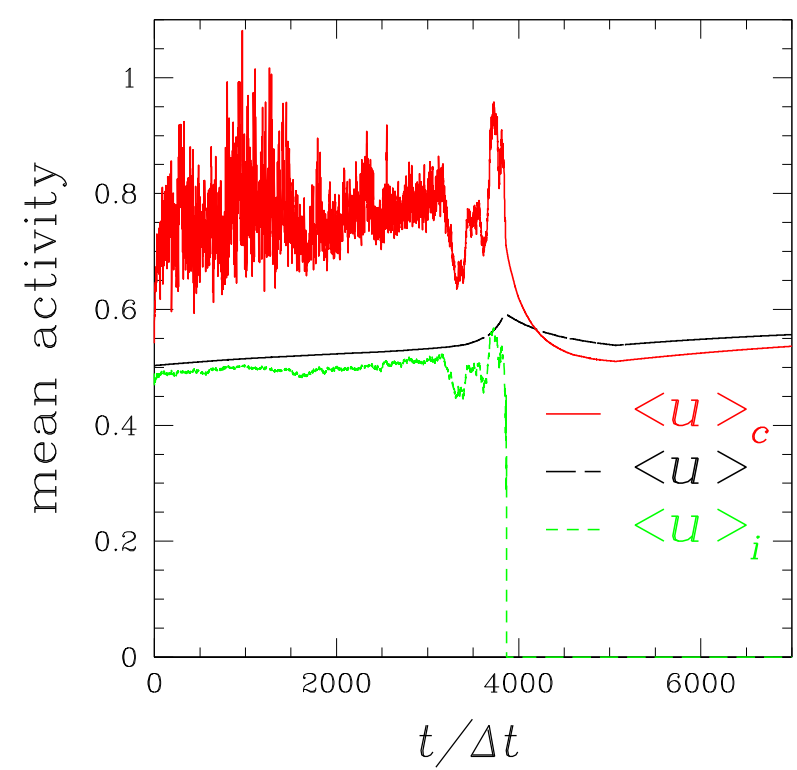

(b)

(c)

FIG. 1. Time evolutions of (a) the numbers of cells $N_{c}$ and $N_{i}$ (the inset plot is a zoom-in on the first time steps), (b) the mean activities $\langle u\rangle,\langle u\rangle_{c}$, and $\langle u\rangle_{i}$, (c) the second moments of the activity $\left\langle u^{2}\right\rangle,\left\langle u^{2}\right\rangle_{c}$, and $\left\langle u^{2}\right\rangle_{i}$. The black long-dashed lines refer to the entire system. The index $c$ refers to cancer cells (red solid lines). The index $i$ refers to immune system cells (green short-dashed lines). Parameter values: $E=10^{-4}, k_{2}=10^{-2}, k_{3}=10^{-3}$.

different types of cells and between the cells and the thermostat are successively performed. The maximum number of binary interactions between a total number $N$ of cells during $\Delta t$ is assessed at $r=N(N-1) k_{\max } \Delta u_{\max } \Delta t$, where $k_{\max }$ is the maximum rate constant among $k_{1}, k_{2}$, and $k_{3}$, and $\Delta u_{\max }$ is the maximum positive difference between the activities $u$ and $u^{\prime}$ of two cells.

First, $r$ interactions between cells are tempted and accepted according to their probability of occurrence. For example, in the case of the process given in Eq. (1), the interaction between a randomly chosen cancer cell $c(u)$ and a randomly chosen normal cell $n\left(u^{\prime}\right)$ is accepted proportionally to $\frac{k_{1}\left(u-u^{\prime}\right)}{k_{\max } \Delta u_{\max }}$ if $u>u^{\prime}$ and rejected otherwise. After an interaction has been accepted, the activities of the interacting cells and the numbers of cells of each type are updated as required by the considered process. The maximum difference of activities, $\Delta u_{\max }$, is also updated. In the particular case of the processes given in Eq. (1), once the mutation of a normal cell $n\left(u^{\prime}\right)$ into a cancer cell $c\left(u^{\prime}\right)$ occurred, a normal cell $n\left(u^{\prime \prime}\right)$ is simultaneously introduced in the system with an activity $u^{\prime \prime}$ randomly chosen according to the probability $P(u)$. Hence, the total number $N_{n}$ of normal cells remains constant.

Then, each cell interacts with the thermostat associated with the field E. Following Eqs. (4) and (5), we perform the 
following update of the activity of each cell at each time step:

$$
u(t+\Delta t)=u(t)+\Delta t E\left(1-\frac{\langle u\rangle}{\left\langle u^{2}\right\rangle} u(t)\right)
$$

where $\langle u\rangle$ and $\left\langle u^{2}\right\rangle$ are the continuously updated mean value and second moment of the activity of the whole system, respectively. It can be noticed that, whereas the interactions between cells listed in Eqs. (1)-(3) may only increase the mean activity $\langle u\rangle$ of the entire system, the thermostat may decrease it when large activity fluctuations of a sufficient number of cells arise. This situation is illustrated in Fig. 1 when the immune system cells suddenly disappear, letting the system with many cancer cells of anomalously large activities: The thermostat rapidly brings their activity down, leading to a decrease of the ensemble average value $\langle u\rangle$. However, in most cases, the mean activity of the entire system has the tendency to increase.

Binary interactions between cells and interactions between the cells and the thermostat are performed successively during a sufficiently long time, which generates a stochastic time series for the number $N_{c}$ of cancer cells, the number $N_{i}$ of immune system cells, the mean value $\langle u\rangle$ of the activity, and the second moment $\left\langle u^{2}\right\rangle$ of the activity. The proposed algorithm aims at directly simulating the kinetic equations given in Eqs. (6)-(9) by additionally reproducing the internal fluctuations inherent to small systems.

\section{RESULTS}

The kinetic Monte Carlo algorithm of thermostatted cell interactions is used to generate stochastic trajectories for different values of the field $E$ associated with the thermostat and different values of the rate constants $k_{2}$ and $k_{3}$ associated with the interactions of a cancer cell and an immune system cell. In all the simulations, we choose the same initial state, defined by the total number of cells $N(t=0)=10^{4}$, the number of cancer cells $N_{c}(t=0)=100$, and the number of immune system cells, $N_{i}(t=0)=100$, at time $t=0$. The number of normal cells remains equal to $N_{n}=9800$ throughout the simulation. We also set $k_{1}=10^{-6}, \mu=0.2$, $\sigma=0.5, \epsilon=10^{-3}$, and $\Delta t=1$, where $k_{1}$ is the rate constant associated with the interaction between a cancer cell and a normal cell given in Eq. (1), $\mu$ and $\sigma$ are the mean value and the standard deviation of the initial distribution of activities, respectively, $\epsilon$ is the activity increase that the cell bearing the largest activity before the interaction inherits after it, and $\Delta t$ is the simulation time step. Depending on the values of the field $E$, characterizing the efficiency of the thermostat, and the rate constants $k_{2}$ and $k_{3}$, associated with the interactions between cancer cells and immune system cells according to Eqs. (2) and (3), different classes of behaviors are observed.

The case of a sufficiently small value of $E$, i.e., a poorly effective thermostat, is first examined. The initial condition imposes the same number of immune system cells and cancer cells and the same distribution of activities between the two populations. Hence, the relative values of $k_{2}$ and $k_{3}$ determine which of the processes given in Eqs. (2) and (3) will initially control the dynamics. Typical results obtained for $k_{2}>k_{3}$ and a small field value $E$ are given in Fig. 1. At the very beginning, the autocatalytic formation of immune system cells through Eq. (2) will be kinetically favored with respect to the autocatalytic formation of cancer cells through Eq. (3). As observed in Fig. 1(a), the number $N_{i}$ of immune system cells is growing rapidly, while the number $N_{c}$ of cancer cells decreases to reach a very low level. This short first period models immunosurveillance and can be called elimination by reference to the terminology introduced in immunoediting. ${ }^{2}$

Then, a long period, during which the number $N_{i}$ of immune system cells slightly increases and the number $N_{c}$ of cancer cells remains quasi-stationary, is observed. This period of slow evolution can be called equilibrium. ${ }^{2}$ However, the condition on the activities associated with the realisation of Eq. (2) imposes that the process takes place only if the interacting cancer cell has a smaller activity than the one of the immune system cell. Consequently, the process given in Eq. (2) most likely results in the withdrawal of the elements with a small activity from the population of the cancer cells. Hence, the remaining cancer cells tend to have a higher mean activity $\langle u\rangle_{c}$ than the mean activity $\langle u\rangle_{i}$ of the immune system cells, as shown in Fig. 1(b). As a result, the activitydependent rate of Eq. (2) decreases whereas the one of Eq. (3) increases. When the numbers of immune system cells and cancer cells exceed a given threshold, of the order of 700 for $N_{i}$ and 150 for $N_{c}$ for the chosen parameter values, the dynamics switches from the control by the process given in Eq. (2) to a control by the process given in Eq. (3). Then, the autocatalytic formation of cancer cells leads to the rapid increase of $N_{c}$ and the rapid decrease of $N_{i}$. But now, Eq. (3) favors the formation of cancer cells with an activity inherited from immune system cells that were selected for their small activity: As $N_{c}$ increases, the mean activity of cancer cells $\langle u\rangle_{c}$ decreases. Consequently, the activity-dependent rate of Eq. (3) decreases and the dynamics is again controlled by Eq. (2). The variations of $N_{c}$ and $\langle u\rangle_{c}$ are anti-correlated as shown in Figs. 1(a) and 1(b). The dependence of the rates on the activities introduces a feedback mechanism, comparable to a feedback loop in a chemical mechanism associated with temporal oscillations and the emergence of a limit cycle in the space of densities. ${ }^{26}$ In the present context and for a poorly efficient thermostat, this phenomenon leads to increasingly large fluctuations of the numbers of cancer cells and immune system cells until one of the fluctuations leads to a vanishing value for $N_{i}$.

When the rate constant $k_{2}$ associated with the autocatalytic formation of immune system cells is larger than the rate constant $k_{3}$ associated with the autocatalytic formation of cancer cells, we have checked that the vanishing of $N_{i}$ and not $N_{c}$ is systematically obtained for different realisations of the stochastic processes. Actually, the increase of $\langle u\rangle_{c}$ due to Eq. (2) offers a decisive advantage to the tumor cells, in relation with the increase of the rate of the process given in Eq. (1), which produces cancer cells faster from normal cells. No equivalent process occurs for the immune system cells that can only be produced by a favorable interaction with cancer cells. Contrary to intuition and due to the complex regulation induced by activity-dependent rates, the condition $k_{2}>k_{3}$ eventually leads to the death of all the immune system cells 
and the proliferation of the cancer cells. The last third period of the evolution shown in Fig. 1(a) illustrates the phenomenon of tumor escape, well known in immunoediting., ${ }^{2,45}$

The bad performances of the thermostat obtained for a small value of the field $E=10^{-4}$ are shown in Fig. 1(c). Tumor escape is associated with an increase of the second moment $\left\langle u^{2}\right\rangle$ of the activity of the entire system that thermalization is not able to prevent. The results shown in Fig. 1 prove that the processes given in Eqs. (1)-(3) successfully include the modeling of the three E's characterizing immunoediting: ${ }^{2} \mathrm{~A}$ fast elimination step, followed by a long equilibrium period and the final tumor escape are clearly illustrated by the evolution of the number of cancer cells and immune system cells given in Fig. 1(a).

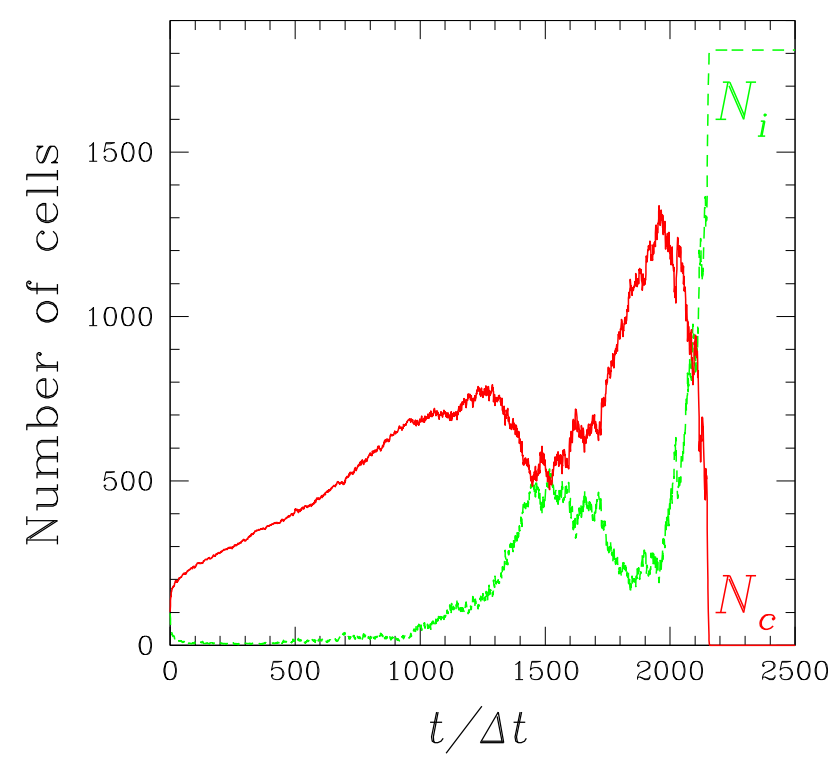

(a)

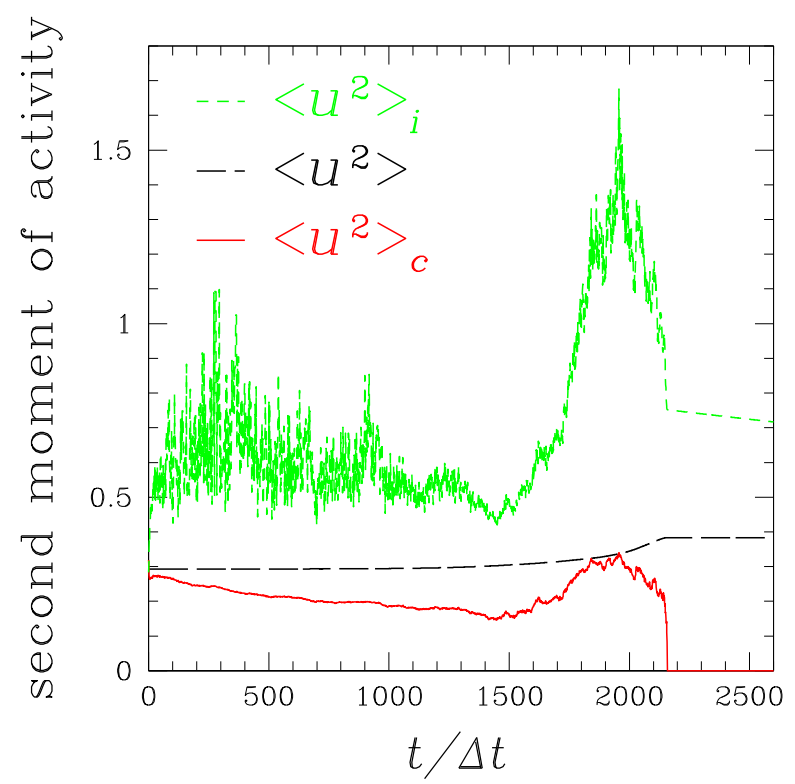

The opposite behavior is observed in Fig. 2 for $k_{2}<k_{3}$, i.e., a rate constant associated with the autocatalytic formation of immune system cells smaller than the rate constant associated with the autocatalytic formation of cancer cells. What was true in Fig. 1 for the cancer cells is observed in Fig. 2 for the immune system cells, and reciprocally. In this case, the autocatalytic production of cancer cells from the normal cells by Eq. (1) is slowed down by the smaller mean activity $\langle u\rangle_{c}$ of the cancer cells. A large fluctuation of the number of cancer cells eventually leads to their complete elimination. Paradoxically, the immune system is able to eradicate the tumor when the rate constant values favor the autocatalytic formation of cancer cells with respect to immune system cells, in relation with the detrimental impact of cancer

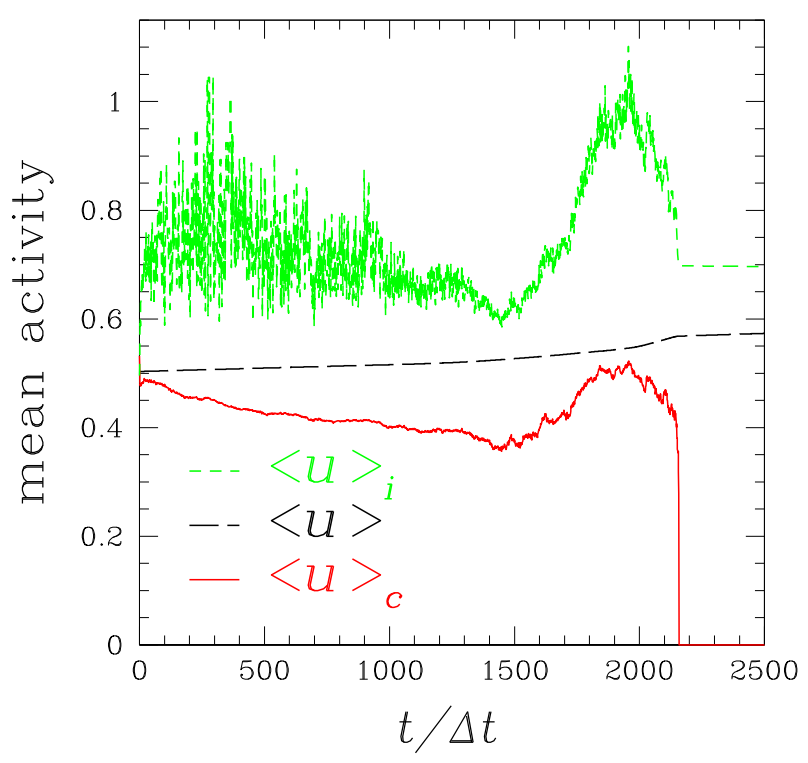

(b)

(c)

FIG. 2. Same caption as in Fig. 1 for $E=10^{-4}, k_{2}=10^{-3}, k_{3}=10^{-2}$. 
cell activity decrease on the kinetics of cancer cell formation from normal cells.

Figure 3 illustrates the exotic behavior obtained for $k_{2}=k_{3}$ when the initial rates of the processes given in Eqs. (2) and (3) are equal. As shown in Fig. 3(a), both $N_{c}$ and $N_{i}$ slowly increase in average and show increasingly large anti-correlated fluctuations, eventually leading to the vanishing of $N_{i}$. In the terminology of immunoediting, the elimination step is absent and the behavior resembles a two-step mechanism composed of equilibrium and tumor escape from the surveillance of the immune system. However, contrary to the distinct behaviors of the mean activities $\langle u\rangle_{c}$ and $\langle u\rangle_{i}$ observed in Fig. 1(b) for $k_{2}>k_{3}$, the two cell populations maintain the same mean activity level, as shown in Fig. 3(b). Consequently, the activity-

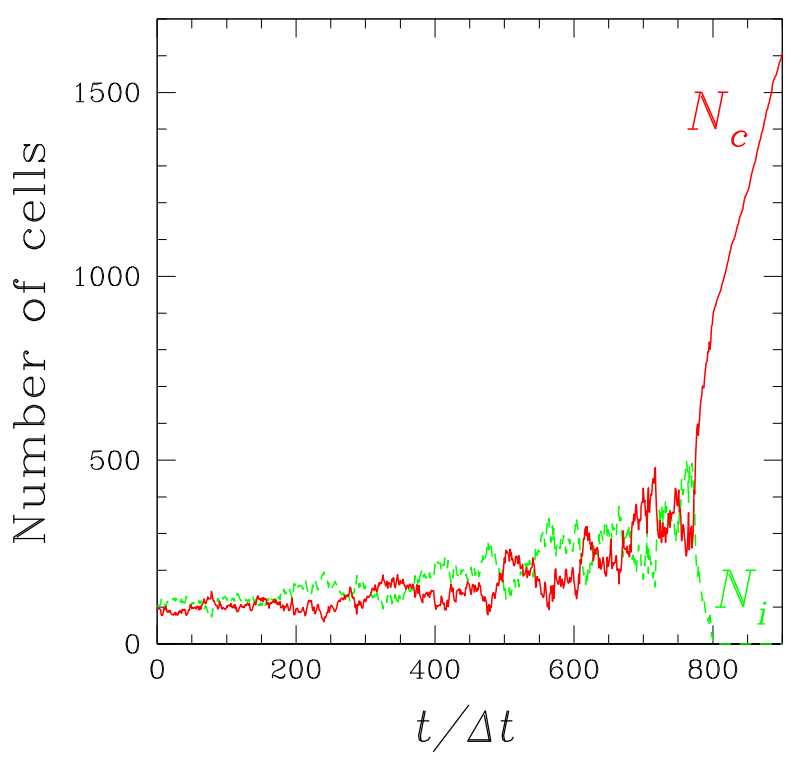

(a)

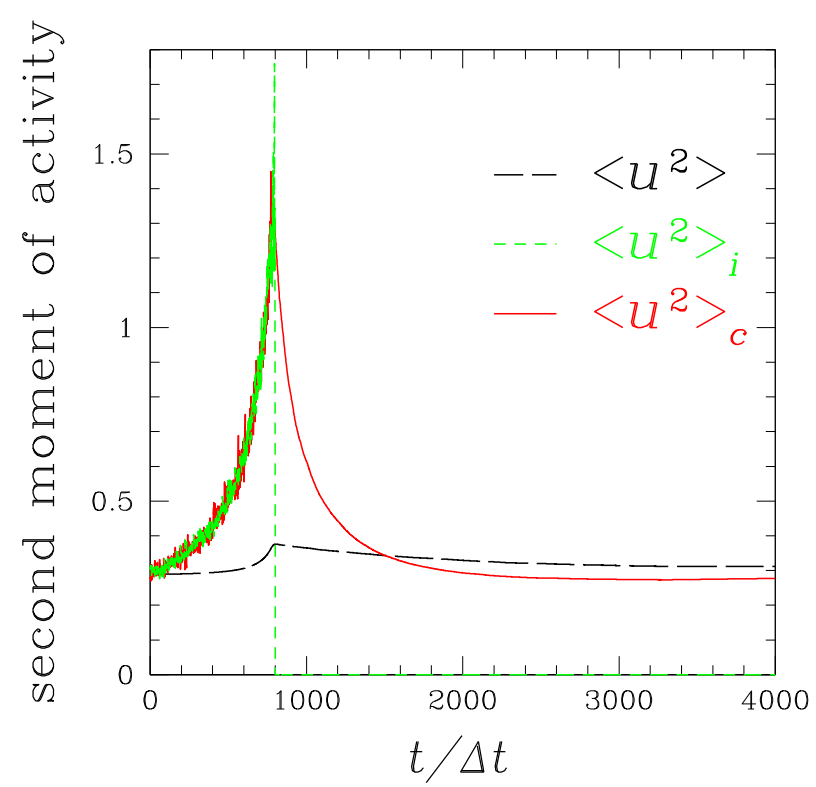

dependent rates associated with the interaction of a cancer cell and an immune system cell remain close throughout the entire evolution. For $k_{2}=k_{3}$, the competition between the two pathways, leading to the proliferation of either immune system cells or cancer cells, is not discriminating. The final increase of the number of cancer cells can be assigned to the autocatalytic production of cancer cells by Eq. (1), kinetically favored by the increase of mean cancer cell activity.

As a partial conclusion, we state that in the absence of efficient thermalization, large fluctuations of cell activity and consequently activity-dependent rates lead to large fluctuations of the number of immune system cells and cancer cells, one of which results in the vanishing of the corresponding cell population. The relative values of the

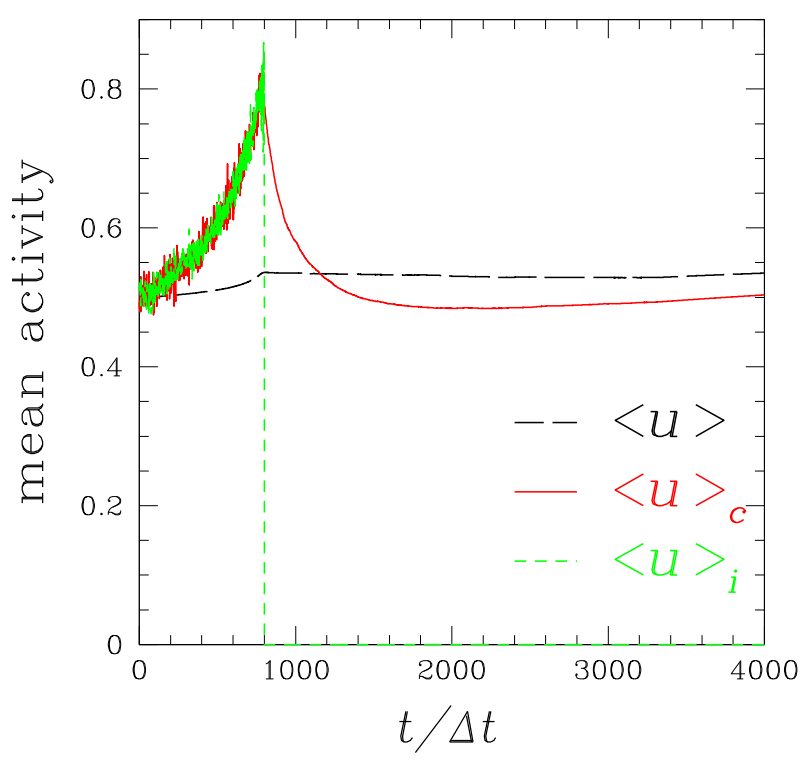

(b)

(c)

FIG. 3. Same caption as in Fig. 1 for $E=10^{-4}, k_{2}=10^{-2}, k_{3}=10^{-2}$. 
rate constants $k_{2}$ and $k_{3}$ associated with the autocatalytic production of either immune system cells or cancer cells, respectively, control the final state of the system. For $k_{2} \geq k_{3}$, i.e., the initial kinetically favorable formation of immune system cells nevertheless leads to tumor escape and proliferation of cancer cells. On the other hand, the condition $k_{2}<k_{3}$, associated with the initial increase of cancer cells, eventually leads to their total elimination. We examine in the next paragraphs how the strength of the thermostat may influence the behavior of the system.

We consider a larger value of the field $E$ associated with the thermostat. As previously shown, different states are eventually reached, depending on the relative values of the rate constants $k_{2}$ and $k_{3}$ but these final states differ from the ones

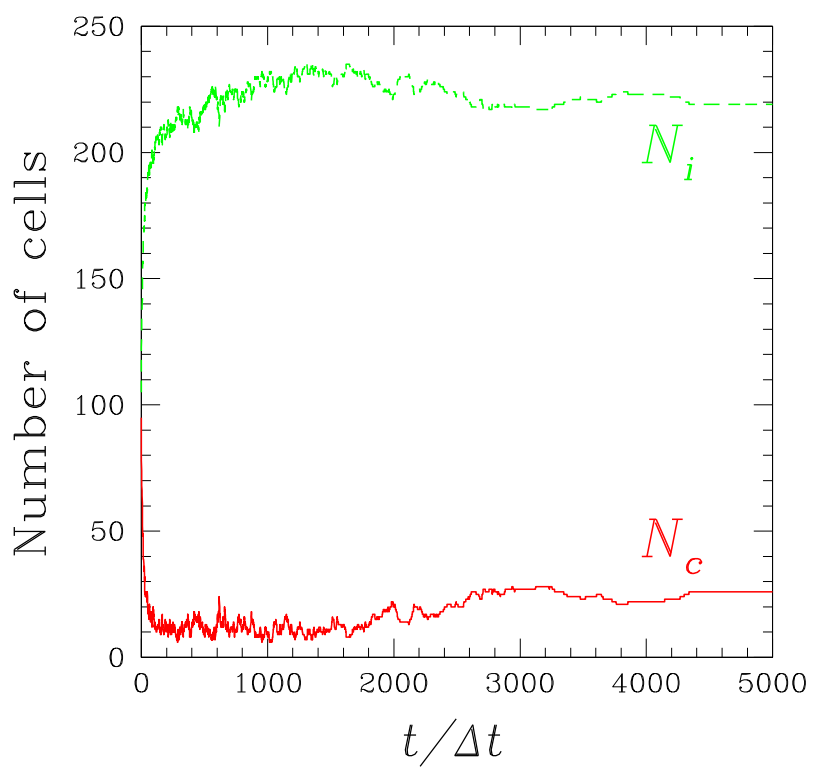

(a)

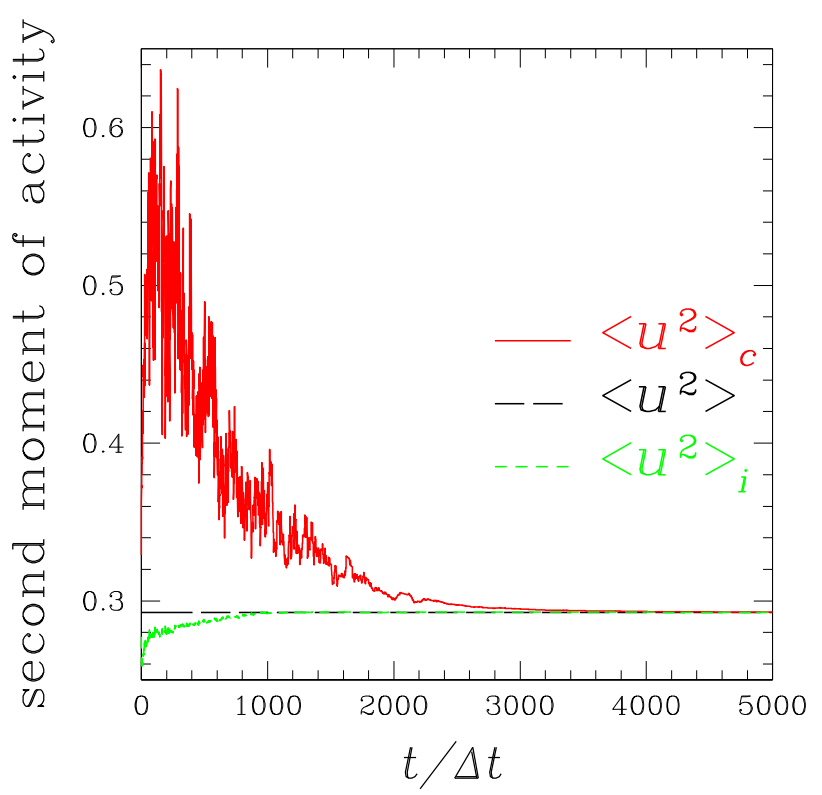

obtained for a smaller value of the field $E$. Figure 4, obtained for $E=10^{-3}$, is to be compared with Fig. 1, obtained for $E$ $=10^{-4}$. The other parameter values are identical. In particular, the rate constants obey: $k_{2}>k_{3}$. In both Figs. 1 and 4 , the immune system cells benefit from an initial advantage, due to their first kinetically favored autocatalytic production through Eq. (2). As already explained and due to the activity-dependent criterion of selection of the encounters in Eq. (2), the initial increase of the number $N_{i}$ of immune system cells is accompanied by a decrease of their mean activity $\langle u\rangle_{i}$ and an increase of cancer cell mean activity $\langle u\rangle_{c}$. Whereas this phenomenon was inducing a feedback mechanism in the case of an inefficient thermostat, the fluctuations of activity and consequently of cell numbers, whose dynamics highly depends on activity,

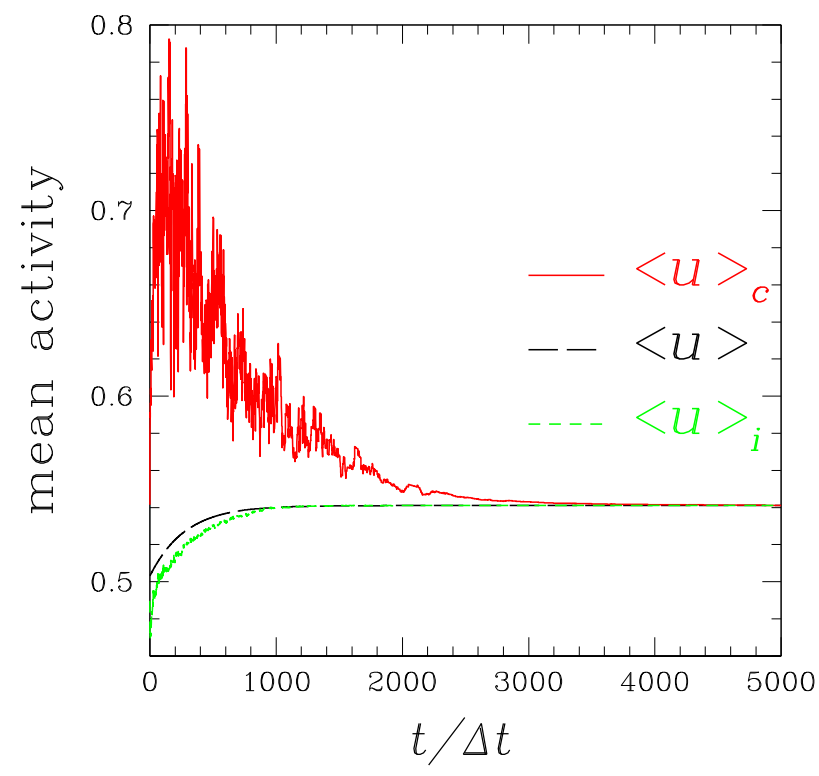

(b)

(c)

FIG. 4. Same caption as in Fig. 1 for $E=10^{-3}, k_{2}=10^{-2}, k_{3}=10^{-3}$. 
are significantly damped for a higher value of the field $E$. Hence, in the presence of a sufficiently effective thermostat, the initially larger production rate of the immune system cells represents a decisive advantage, leading to the stabilisation of the system in a stationary state with a final number of immune system cells larger than the final number of cancer cells.

The results given in Fig. 5, to be compared with Fig. 2, are obtained for $k_{2}<k_{3}$. Again, a sufficient thermalization dampens the fluctuations of the number of cancer cells and immune system cells. Hence, the initial increase of the number of cancer cells prevails in the long term. For $k_{2}<k_{3}$, the system reaches a nonequilibrium steady state with a number of immune system cells smaller than the number of cancer cells. Only the specific case $k_{2}=k_{3}$ qualitatively leads to the

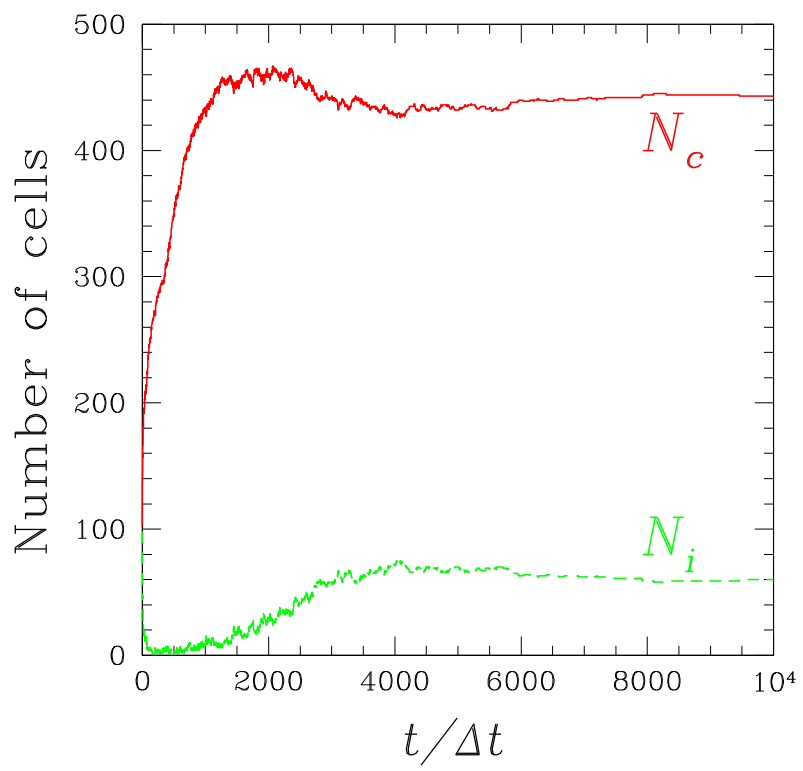

(a)

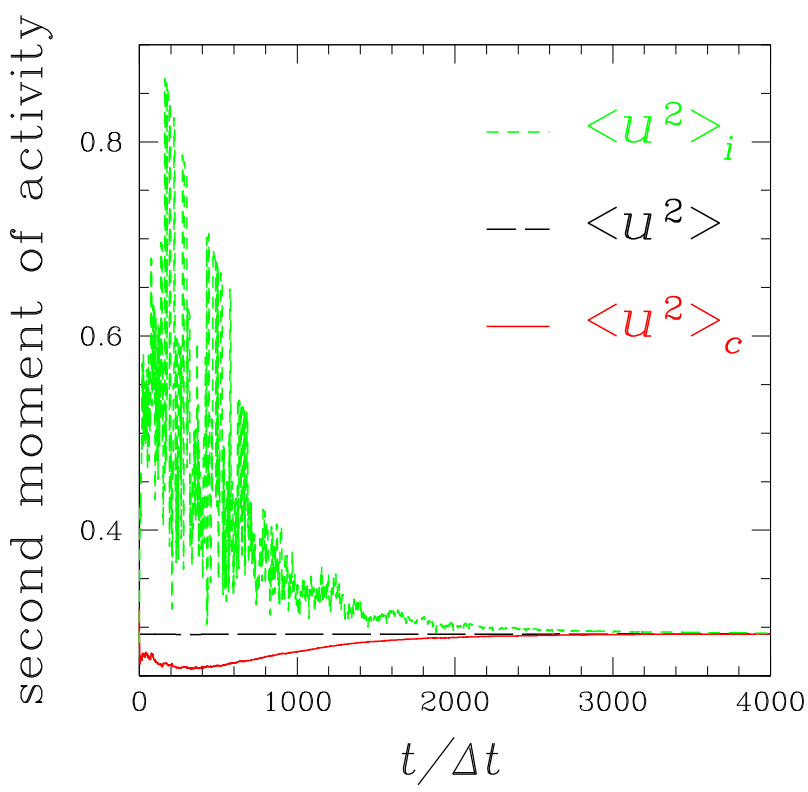

same results for $E=10^{-3}$ as for $E=10^{-4}$. A larger field $E$ does not succeed in suppressing the fluctuations of $N_{i}$ and $N_{c}$ and tumor escape is equally observed.

Hence, the results obtained in the case of a sufficiently effective control of activity fluctuations are more in line with intuition. For different values of the rate constants $k_{2}$ and $k_{3}$ which control the dynamics of the interactions between the immune system cells and the cancer cells, the system reaches a stationary state associated with nonvanishing values of both the number $N_{c}$ of cancer cells and $N_{i}$ of immune system cells. A smaller stationary value of the number of cancer cells is obtained for $k_{3}<k_{2}$, i.e., in the case of kinetically disadvantaged cancer cell formation.

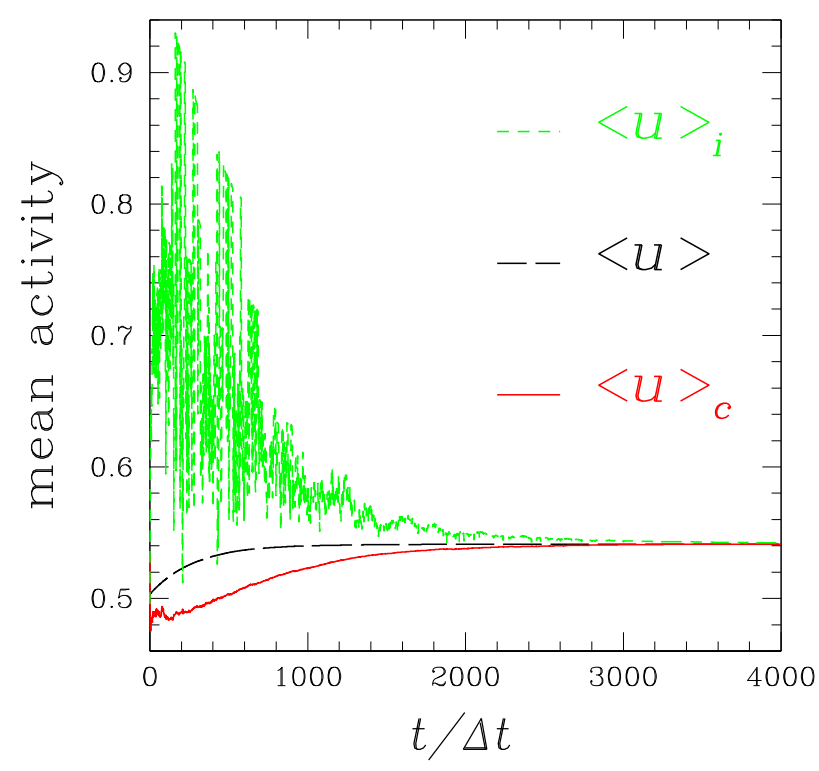

(b)

(c)

FIG. 5. Same caption as in Fig. 1 for $E=10^{-3}, k_{2}=10^{-3}, k_{3}=10^{-2}$. 


\section{CONCLUSION}

In order to model cancer immunosurveillance at cell scale, we propose a minimal model of binary interactions between three types of cells, normal cells, cancer cells, and immune system cells. The model of interactions resembles a chemical mechanism including autocatalytic steps. The analogy with open chemical systems, maintained far from equilibrium due to exchanges with a reservoir, is also used to mimic the production of normal cells by living tissues. The cells are supposed to follow a strategy leading to the increase of a nonconserved quantity called activity. The cancer cells and immune system cells are produced with activity-dependent rates, which induces a feedback loop, responsible for large random oscillations of the cell numbers. Cell interactions are described in the framework of thermostatted kinetic theory.

We have developed a kinetic Monte Carlo algorithm consisting of the succession of binary cell interactions and thermalization of cell activity. In the case of a poorly efficient thermostat, i.e., for large fluctuations of the activities and the numbers of cells, the model successfully reproduces the three E's of immunoediting (elimination, equilibrium, and escape) for nonintuitive values of the dynamical parameters. The feedback mechanism plays a crucial role in eventual tumor escape, observed for the initially kinetically favored production of immune system cells. In the case of a better thermalization, the system is stabilized in a nonequilibrium steady state with nonvanishing values of both the number $N_{c}$ of cancer cells and the number $N_{i}$ of immune system cells. More intuitive results are obtained, with larger final values of $N_{i}$ for a larger rate constant associated with the autocatalytic production of immune system cells and reciprocally, larger final values of $N_{c}$ for a larger rate constant associated with the autocatalytic production of cancer cells.

In the model, the heterogeneity of cancer cells is taken into account through the distribution of the activities but the considered system is spatially homogeneous. The kinetic
Monte Carlo approach can be generalized to inhomogeneous distributions of cells. Work in this direction is certainly desirable, in order to account for the spatial spreading of a tumor and for taking into account the role of metastasis.

${ }^{1}$ T. Blankenstein, P. G. Coulie, E. Gilboa, and E. M. Jaffee, Nat. Rev. Cancer 12, 307 (2012).

${ }^{2}$ G. P. Dunn, A. T. Bruce, H. Ikeda, L. J. Old, and R. D. Schreiber, Nat. Immunol. 3, 991 (2002).

${ }^{3}$ F. H. Igney and P. H. Krammer, J. Leukoc. Biol. 71, 907 (2002).

${ }^{4}$ G. P. Dunn, L. J. Old, and R. D. Schreiber, Annu. Rev. Immunol. 22, 329 (2004).

${ }^{5}$ M. D. Vesely and R. D. Schreiber, Ann. N. Y. Acad. Sci. 1284, 1 (2013).

${ }^{6}$ C. Bianca and C. Dogbe, Nonlinearity 27, 2771 (2014).

${ }^{7}$ C. Bianca and A. Lemarchand, Commun. Nonlinear Sci. Numer. Simul. 20, 14 (2015).

${ }^{8}$ C. Bianca, C. Dogbe, and A. Lemarchand, Acta Appl. Math. 189, 1 (2015).

${ }^{9}$ M. C. Marcetti, J. F. Joanny, S. Ramaswamy, T. B. Liverpool, J. Prost, M. Rao, and R. Adita Simha, Rev. Mod. Phys. 85, 1143 (2012).

${ }^{10}$ B. Wennberg and Y. Wondmagegne, J. Stat. Phys. 124, 859 (2006).

${ }^{11}$ C. Bianca, Nonlinear Anal.: Real World Appl. 13, 2593 (2012).

${ }^{12}$ C. Cercignani, R. Illner, and M. Pulvirenti, The Mathematical Theory of Dilute Gases (Springer, New York, 1994).

${ }^{13}$ C. Cercignani, "Recent results in the kinetic theory of granular materials," in Perspectives and Problems in Nonlinear Science, edited by E. Kaplan, J. E. Marsden, and K. R. Sreenivasan (Springer, New York, 2003), pp. 217-228.

${ }^{14}$ J. J. Brey, J. W. Dufty, C. S. Kim, and A. Santos, Phys. Rev. E 58, 4638 (1998).

${ }^{15}$ J. M. Montanero and A. Santos, Granular Matter 2, 53 (2000).

${ }^{16}$ G. A. Bird, Molecular Gas Dynamics and the Direct Simulation of Gas Flows (Clarendon, Oxford, 1994).

${ }^{17}$ D. T. Gillespie, Annu. Rev. Phys. Chem. 58, 35 (2007).

${ }^{18}$ F. Alexander and A. Garcia, Comput. Phys. 11, 588 (1997).

${ }^{19}$ B. Nowakowski and A. Lemarchand, Phys. Rev. E 62, 3156 (2000).

${ }^{20}$ B. Nowakowski and A. Lemarchand, Phys. Rev. E 68, 031105 (2003).

${ }^{21}$ P. Dziekan, A. Lemarchand, and B. Nowakowski, J. Chem. Phys. 137, 074107 (2012).

${ }^{22}$ S. Yasuda, preprint arXiv:1503.08099 (2015).

${ }^{23}$ C. W. Gardiner, Handbook of Stochastic Methods (Springer, Berlin, 1985).

${ }^{24} \mathrm{M}$. Kac, in The Boltzmann Equation, Theory and Applications, The Series Acta Physica Austriaca Vol. 10, edited by E. G. D. Cohen and W. Thirring (Springer, Vienna, 1973), pp. 379-400.

${ }^{25}$ W. Wagner, J. Stat. Phys. 66, 1011 (1992).

${ }^{26}$ G. Nicolis and I. Prigogine, Self-Organization in Nonequilibrium Systems (Wiley, New York, 1977). 